\title{
Multiferroic Magnetic Spirals Induced by Random Magnetic Exchanges
}

\author{
Andrea Scaramucci, ${ }^{1,2, *}$ Hiroshi Shinaoka, ${ }^{3,4,8, \dagger}$ Maxim V. Mostovoy, ${ }^{5}$ Markus Müller, ${ }^{6,7}$ Christopher Mudry, ${ }^{6}$ \\ Matthias Troyer, ${ }^{3,9}$ and Nicola A. Spaldin ${ }^{2}$ \\ ${ }^{1}$ Laboratory for Scientific Development and Novel Materials, Paul Scherrer Institut, \\ 5235, Villigen PSI, Switzerland \\ ${ }^{2}$ Materials Theory, ETH Zurich, CH-8093 Zürich, Switzerland \\ ${ }^{3}$ Institute for Theoretical Physics, ETH Zurich, CH-8093 Zürich, Switzerland \\ ${ }^{4}$ Department of Physics, University of Fribourg, 1700 Fribourg, Switzerland \\ ${ }^{5}$ Zernike Institute for Advanced Materials, University of Groningen, Nijenborgh 4, 9747 AG, Groningen, \\ Netherlands \\ ${ }^{6}$ Condensed Matter Theory Group, Paul Scherrer Institute, CH-5232 Villigen PSI, Switzerland \\ ${ }^{7}$ The Abdus Salam International Centre for Theoretical Physics, 34151 Trieste, Italy \\ ${ }^{8}$ Department of Physics, Saitama University, Saitama 338-8570, Japan \\ ${ }^{9}$ Microsoft Research, Redmond, Washington 98052, USA
}

(Received 20 December 2016; revised manuscript received 23 October 2017; published 10 January 2018)

\begin{abstract}
Multiferroism can originate from the breaking of inversion symmetry caused by magnetic-spiral order. The usual mechanism for stabilizing a magnetic spiral is competition between magnetic exchange interactions differing by their range and sign, such as nearest-neighbor and next-nearest-neighbor interactions. In insulating compounds, it is unusual for these interactions to be both comparable in magnitude and of a strength that can induce magnetic ordering at room temperature. Therefore, the onset temperatures for multiferroism through this mechanism are typically low. By considering a realistic model for multiferroic $\mathrm{YBaCuFeO}_{5}$, we propose an alternative mechanism for magnetic-spiral order, and hence for multiferroism, that occurs at much higher temperatures. We show, using Monte Carlo simulations and electronic structure calculations based on density functional theory, that the Heisenberg model on a geometrically nonfrustrated lattice with only nearest-neighbor interactions can have a spiral phase up to high temperature when frustrating bonds are introduced randomly along a single crystallographic direction as caused, e.g., by a particular type of chemical disorder. This long-range correlated pattern of frustration avoids ferroelectrically inactive spin-glass order. Finally, we provide an intuitive explanation for this mechanism and discuss its generalization to other materials.
\end{abstract}

DOI: 10.1103/PhysRevX.8.011005

\section{INTRODUCTION}

Insulators with magnetic spiral order are of particular interest because of their associated multiferroism [1-5] in which the breaking of inversion symmetry by the magnetic spiral drives long-range ferroelectric order. The magnetic order can then be manipulated by an applied voltage with minimal current dissipation because of the insulating nature offering potential for low-loss memory devices.

For many insulators, such as the orthorhombic manganites $R \mathrm{MnO}_{3}(R=\mathrm{Dy}, \mathrm{Tb})[6-10]$, spiral order results from a

\footnotetext{
*andrea.scaramucci@gmail.com

†shinaoka@itp.phys.ethz.ch
}

Published by the American Physical Society under the terms of the Creative Commons Attribution 4.0 International license. Further distribution of this work must maintain attribution to the author(s) and the published article's title, journal citation, and DOI.
Subject Areas: Condensed Matter Physics, Magnetism, Materials Science competition between nearest-neighbor and further-neighbor magnetic exchanges of comparable strength. On the one hand, in magnetic lattices without geometric frustration, it is rare that these interactions are both comparable in magnitude and of the scale of room temperature. A similar strength of nearestneighbor and further-neighbor magnetic exchanges is more frequently found when they have relatively small values. As a consequence, the onset temperature of the spiral state, $T_{\mathrm{spi}}$, is rather low, strongly limiting the multiferroic temperature range. On the other hand, geometrically frustrated lattices can have relatively large competing exchanges of similar magnitude. However, in these compounds, the strong frustration drastically lowers the magnetic transition temperature. Alternative routes to stabilizing magnetic spirals at higher temperatures in magnetic lattices without geometric frustration are, therefore, of fundamental and technological interest.

The phenomenology of the spiral magnet $\mathrm{YBaCuFeO}_{5}$, which has one of the highest critical temperatures among 
the magnetically driven multiferroics $[11,12]$, suggests that a particular type of chemical disorder might provide such a route. As the temperature is lowered below $T_{\mathrm{AF}} \sim 440 \mathrm{~K}$ in $\mathrm{YBaCuFeO}_{5}$, the paramagnetic state undergoes a transition to a commensurate magnetic order with wave vector $\boldsymbol{q}_{\mathrm{AF}}=(1 / 2,1 / 2,1 / 2)$. Then, below $T_{\mathrm{spi}}<T_{\mathrm{AF}}$, a multiferroic magnetic spiral phase sets in, with a propagation wave vector along the $c$ crystallographic axis, $\boldsymbol{q}_{\mathrm{spi}}=(1 / 2,1 / 2,1 / 2-Q)$. The value of $Q$ increases smoothly from $Q\left(T_{\text {spi }}\right)=0$ as temperature is decreased. Importantly, the reported values of $T_{\text {spi }}$ range from $180 \mathrm{~K}$ to $310 \mathrm{~K}$ [11-16] depending on the preparation conditions, and it was recently shown [16] that $T_{\text {spi }}$ and $Q$ increase systematically with $\mathrm{Fe}^{3+} / \mathrm{Cu}^{2+}$ occupational disorder. These observations suggest that chemical disorder plays an essential role in stabilizing the magnetic spiral motivating our search for a microscopic mechanism by which disorder facilitates, or even drives, magnetic spiral order.

In this paper, we introduce a classical Heisenberg spin model for $\mathrm{YBaCuFeO}_{5}$ in which spiral order is indeed induced by chemical disorder. We describe the local moments of $\mathrm{Cu}^{2+}$ and $\mathrm{Fe}^{3+}$ as classical Heisenberg spins, $\boldsymbol{S}_{\boldsymbol{r}}$, localized at the positions $\boldsymbol{r}$ of a lattice. We assume only nearest-neighbor exchange interactions and use the magnitudes calculated from the local spin-density approximation, including an effective Hubbard $U$ correction $(\mathrm{LSDA}+U)$ for $\mathrm{YBaCuFeO}_{5}$ [17]. Without chemical disorder, the magnet is unfrustrated and establishes commensurate antiferromagnetic order at $T_{\mathrm{AF}} \approx 300 \mathrm{~K}$.
Frustration is introduced through dilute impurity bonds with enhanced exchange couplings of opposite sign. The structure of $\mathrm{YBaCuFeO}_{5}$ is such that the chemical disorder introduces only collinear impurity bonds parallel to the $c$ axis. We show that the induced frustration results in a local canting of the antiferromagnetic order parameter around the impurity bond, which spontaneously breaks the local inversion symmetry around each impurity so that $S_{r_{\text {imp }}+\delta r} \neq S_{r_{\text {imp }}-\delta r}$. The Goldstone modes of the antiferromagnet mediate a longrange coupling between the cantings of different impurities and establish long-range order of the local cantings that induces a continuous twist of the antiferromagnetic order parameter in the direction parallel to the impurity bonds. We note that randomly oriented impurity bonds would have a spin-glass solution [18-21], whose magnetic order does not couple to a net electric polarization and thus does not lead to multiferroism. This mechanism results in a $T_{\text {spi }}$ of the order of a typical exchange coupling. Our Monte Carlo simulations for $\mathrm{YBaCuFeO}_{5}$ yield $T_{\text {spi }}$ as high as $250 \mathrm{~K}$, depending on the concentration and strength of the impurity bonds, in a manner that is consistent with the experimentally observed dependence of $T_{\text {spi }}$ and $\boldsymbol{q}_{\text {spi }}$ on the amount of $\mathrm{Fe}^{3+} / \mathrm{Cu}^{2+}$ occupational disorder [16].

\section{MICROSCOPIC ORIGIN OF SPIN-SPIRAL STATE IN $\mathrm{YBaCuFeO}_{5}$}

$\mathrm{YBaCuFeO}_{5}$ forms a vacancy-ordered perovskite structure in which planes of $\mathrm{Y}$ ions separate bilayers of $\mathrm{BaCuFeO}_{5}$. The

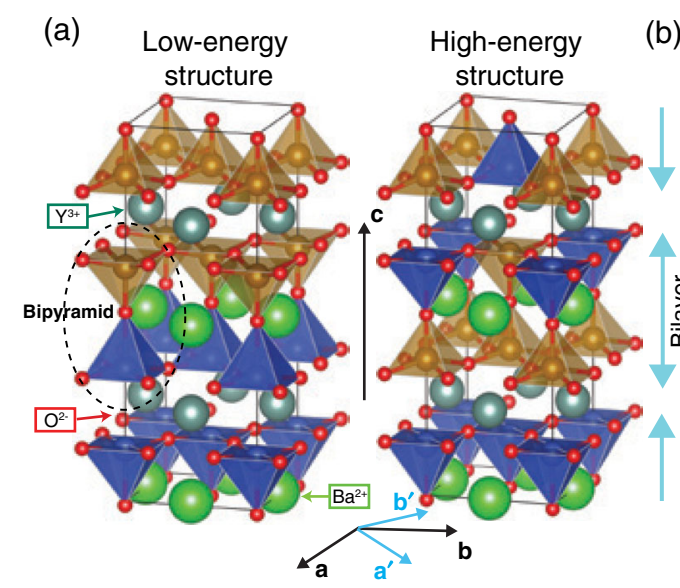

(b) Collinear magnetic
order

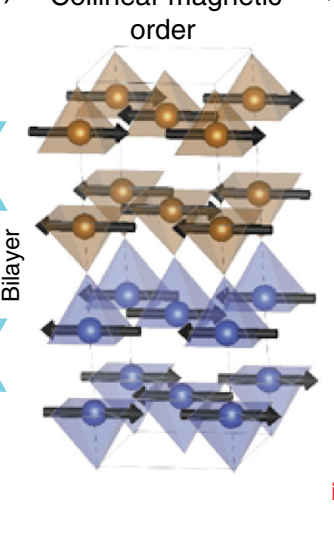

(c)

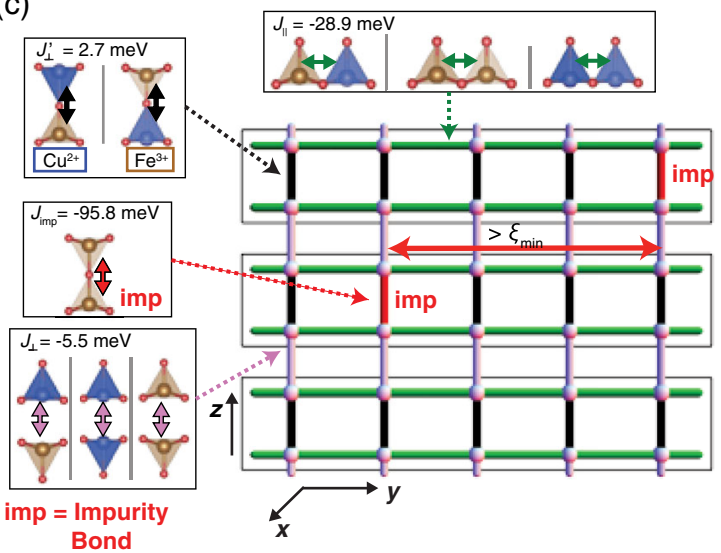

FIG. 1. (a) Two different orderings of $\mathrm{Cu}^{2+}$ and $\mathrm{Fe}^{3+}$ in the $\sqrt{2} \times \sqrt{2} \times 2$ supercell of $\mathrm{YBaCuFeO}_{5}$. The $\mathrm{FeO}_{5}$ and the $\mathrm{CuO}_{5}$ square pyramids are shown in gold and blue, respectively. The left panel shows a low-energy structure consisting only of $\mathrm{Fe}^{3+}-\mathrm{Cu}^{2+}$ bipyramids. The right panel shows a high-energy structure with one $\mathrm{Fe}^{3+}-\mathrm{Fe}^{3+}$ and, upon periodic repetition, one $\mathrm{Cu}^{2+}-\mathrm{Cu}^{2+}$ bipyramid. (b) Unfrustrated magnetic order in the commensurate phase. (c) Simplified spin model for $\mathrm{YBaCuFeO}_{5}$ with nearestneighbor exchange interactions. The $a^{\prime}$ and $b^{\prime}$ axes are rotated by $45^{\circ}$ with respect to the $a$ and $b$ axes in diagram (a). The magnetic ions corresponding to either $\mathrm{Cu}^{2+}$ or $\mathrm{Fe}^{3+}$ are depicted as pink spheres. They form bilayers (regions enclosed by the rectangles) obtained by stacking a pair of $x-y$ square lattices (corresponding to $\mathrm{YBaCuFeO}_{5}$ bilayers) along the $z$ axis. The antiferromagnetic exchange coupling $J_{\|}$within a layer is assumed to be independent of whether it couples $\mathrm{Fe}^{3+}$ with $\mathrm{Fe}^{3+}, \mathrm{Cu}^{2+}$ with $\mathrm{Cu}^{2+}$, or $\mathrm{Fe}^{3+}$ with $\mathrm{Cu}^{2+}$. The interlayer coupling $J_{\perp}^{\prime}$ within a bilayer is ferromagnetic for $\mathrm{Cu}^{2+}-\mathrm{Fe}^{3+}$ bipyramids (see text). Impurity bonds have a strong antiferromagnetic exchange, $J_{\text {imp }}$, similar in magnitude to the ab initio value for $\mathrm{Fe}^{3+}-\mathrm{Fe}^{3+}$ bipyramids. The impurity bonds are randomly distributed, up to the constraint that two impurity bonds in adjacent bilayers cannot be closer than a minimal distance $\xi_{\min }$. 
latter consist of corner-sharing $\mathrm{FeO}_{5} / \mathrm{CuO}_{5}$ bipyramids, as depicted in Fig. 1(a).

A recent $a b$ initio study [12] revealed that the lowestenergy arrangements of $\mathrm{Fe}$ and $\mathrm{Cu}$ ions contain only $\mathrm{Fe}^{3+}-\mathrm{Cu}^{2+}$ bipyramids as shown in the left panel of Fig. 1(a). The exchange interactions between the magnetic ions making up these low-energy structures were also calculated using LSDA $+U$. The exchange interactions within the $\mathrm{Fe}-\mathrm{Cu}$ bipyramids were found to be uniform in sign (ferromagnetic) and so unfrustrated, and thus cannot explain the emergence of a magnetic spiral. Bipyramids of $\mathrm{Cu}^{2+}-\mathrm{Cu}^{2+}$ and $\mathrm{Fe}^{3+}-\mathrm{Fe}^{3+}$, shown in the right panel of Fig. 1(a), are energetically more costly, but they nevertheless occur as local defects that form during preparation and thermal treatment of the sample. The $\mathrm{Fe}^{3+}-\mathrm{Fe}^{3+}$ bipyramids introduce strongly frustrating antiferromagnetic couplings along the $c$ axis [22]. In the following, we show that a small concentration of $\mathrm{Fe}^{3+}-\mathrm{Fe}^{3+}$ bipyramids is at the microscopic origin of the spiral order.

We model the magnetic ordering of $\mathrm{YBaCuFeO}_{5}$ using the classical Hamiltonian

$$
H:=-\frac{1}{2} \sum_{r, r^{\prime}} J_{r, r^{\prime}} S_{r} \cdot S_{r^{\prime}}+H_{\mathrm{SOC}} .
$$

Here, the classical spin $S_{r}$ is a three-component unit vector located at the site $\boldsymbol{r}$ of a cubic lattice isomorphic to the tetragonal lattice of $\mathrm{YBaCuFeO}_{5}$. To distinguish between the lattice vectors of the real material and those of the spin model, we label the latter as $\boldsymbol{x}, \boldsymbol{y}$, and $\boldsymbol{z}$. Their length is equal to $a^{\prime}=b^{\prime} \approx c / 2$, where $\boldsymbol{a}^{\prime}, \boldsymbol{b}^{\prime}$, and $\boldsymbol{c}$ are the lattice vectors of $\mathrm{YBaCuFeO}_{5}$ as shown in Fig. 1 (with $\boldsymbol{c} \| z$ ). To facilitate comparison with experiments, we express the wave vectors in units of the reciprocal vectors of the crystallographic unit cell of $\mathrm{YBaFeCuO}_{5}$ throughout. We retain only four nearestneighbor exchange couplings $J_{r, r^{\prime}} \in\left\{J_{\|}, J_{\perp}^{\prime}, J_{\perp}, J_{\text {imp }}\right\}$. The $a b$ planes host spins on a square lattice, coupled antiferromagnetically with the strong exchange $J_{\|}=-28.9 \mathrm{meV}$ [23]. These planes are stacked along the $c$ axis in bilayers (Fig. 1). Within a bilayer, the nearest neighbors along the $c$ axis, that is, the spins in a bipyramid, are coupled with the weak ferromagnetic exchange interaction $J_{\perp}^{\prime}=$ $2.7 \mathrm{meV}>0$. Adjacent bilayers are coupled with the relatively weak antiferromagnetic coupling $J_{\perp}=-5.5 \mathrm{meV}$. Finally, the defect bipyramids are modeled by a small concentration, $n_{\text {imp }}$, of randomly located "impurity bonds" lying within the bilayers. They substitute $J_{\perp}^{\prime}>0$ with the strong antiferromagnetic coupling $J_{\text {imp }}=-95.8 \mathrm{meV}$ calculated for $\mathrm{Fe}^{3+}-\mathrm{Fe}^{3+}$ bipyramids and locally frustrate the ferromagnetic intrabilayer couplings inducing a local canting for $\left|J_{\text {imp }}\right|$ sufficiently larger than $J_{\|}$. At the same time, we assume impurities to be sufficiently dilute, $\left(n_{\text {imp }}\left|J_{\text {imp }}\right| \lesssim J_{\perp}^{\prime}\right)$, so that intralayer antiferromagnetic alignment does not become favored within bilayers. The impurity bonds are long-range correlated in the sense that they are always oriented parallel to the $c$ axis. We do not include the $\mathrm{Cu}^{2+}-\mathrm{Cu}^{2+}$ bipyramids (which stoichiometry implies to be as abundant as the $\mathrm{Fe}^{3+}-\mathrm{Fe}^{3+}$ bipyramids) since their interlayer exchange is substantially smaller than all other couplings.

The second term in Eq. (1), $H_{\text {SOC }}$, contains terms with a spin-orbit origin. These are expected to be smaller than the exchange couplings for $3 d$ transition metals. These terms explicitly break the spin-rotational symmetry of the Heisenberg exchange interactions. Whereas it is the dominant Heisenberg exchange interactions that are responsible for the existence of the spiral phase in the first place, it is the subdominant $H_{\mathrm{SOC}}$ that selects a preferred orientation of the magnetic spiral plane relative to the crystallographic axis. We note that, in the current experimental literature, there is some debate about the orientation of the magnetic spiral plane. While neutron-scattering investigations in Refs. [12,16] favor a temperature-dependent tilting of the spiral plane with respect to the crystallographic $a b$ plane, the refinements reported by Lai et al. [24] favor the $a b$ plane as the plane of the magnetic spiral at all temperatures. As the main purpose of this paper is to provide a mechanism explaining the origin of the magnetic spiral phase in $\mathrm{YBaCuFeO}_{5}$, we consider the simplest possible form for the second term in Eq. (1), namely,

$$
H_{\mathrm{SOC}}:=\frac{\Delta}{2} \sum_{r}\left(\boldsymbol{S}_{\boldsymbol{r}} \cdot \hat{\boldsymbol{c}}\right)^{2},
$$

and set $\Delta=0.5 \mathrm{meV}$. Note that only the sign of $\Delta$ matters for what follows.

The presence of a spiral phase supported by some given configuration $\left\{\boldsymbol{S}_{\boldsymbol{r}}\right\}$ is signaled by the order parameter

$$
\psi:=\max _{\hat{n}} \psi(\hat{\boldsymbol{n}}),
$$

where the pseudoscalar

$$
\psi(\hat{\boldsymbol{n}}):=\frac{1}{N} \sum_{r}\left(\boldsymbol{S}_{\boldsymbol{r}} \wedge \boldsymbol{S}_{\boldsymbol{r}+z}\right) \cdot \hat{\boldsymbol{n}}
$$

measures the deviation of colinearity between nearestneighbor spins along the $z$ direction projected along the unit vector $\hat{\boldsymbol{n}}$ and averaged over the number $N$ of spins in the lattice.

Note that $\psi$ is independent of the spiral plane favored by the spin-orbit coupling $H_{\text {SOC }}$. The latter merely selects the vector $\hat{\boldsymbol{n}}^{*}$ that maximizes Eq. (3b). For spin-orbit terms of the form (2), one has $\hat{\boldsymbol{n}}^{*} \propto \boldsymbol{c}$. However, as we suggest in Sec. III, alternative, more realistic versions of $H_{\mathrm{SOC}}$ do select a $\hat{\boldsymbol{n}}^{*}$ with a nontrivial tilt angle with respect to the $c$ axis and thus favor a tilt of the magnetic spiral plane out of the $a b$ plane. In the presence of such a tilt, the magnetic spiral order induces an electric polarization $\boldsymbol{P}$. In our case, where frustrating couplings are restricted to bonds along the $c$ axis, up to a multiplicative coupling constant, the polarization is given by [25] 


$$
\boldsymbol{P} \propto \frac{1}{N} \sum_{r}\left(S_{r} \wedge S_{r+z}\right) \wedge \hat{\boldsymbol{c}}=\psi \hat{\boldsymbol{n}}^{*} \wedge \hat{\boldsymbol{c}}
$$

In our Monte Carlo simulations, we use a cubic superlattice of linear dimension $L \times L \times 2 L$ (where $L \leq 28$ ) with periodic boundary conditions in plane and open boundary conditions in the $z$ direction. Impurity bonds are randomly located, but they are subject to the constraint that any two impurity bonds in adjacent bilayers are further apart than the in-plane minimum distance $\xi_{\min }$ (tuned to be 2.5 or 4 in units of the lattice spacing $a^{\prime}=b^{\prime}$ ). This shortdistance cutoff embodies the main effect of the strong Coulomb repulsion between $\mathrm{Fe}^{3+}-\mathrm{Fe}^{3+}$ bipyramids. We consider small impurity bond concentrations $n_{\text {imp }} \leq 0.04 \sim$ $J_{\perp}^{\prime} /\left|J_{\text {imp }}\right|$ per unit cell. Monte Carlo results include a disorder averaging over 16 configurations of the impurity bonds. A more complete description of the model, the method, and detailed Monte Carlo data, including systemsize dependence, are given in Ref. [26].

For the clean, unfrustrated case $\left(n_{\text {imp }}=0\right)$, we find a transition at $T_{\mathrm{AF}} \simeq 300 \mathrm{~K}$, a temperature of the order of $J_{\|}$, from the high-temperature paramagnetic phase to a lowtemperature collinear antiferromagnetic phase with magnetic order parameter $m_{\mathrm{AF}}$ (see definition in Ref. [26]) and ordering wave vector $\boldsymbol{q}_{\mathrm{AF}}=(1 / 2,1 / 2,1 / 2)$. This is consistent with the antiferromagnetic ordering observed experimentally at high temperatures in Ref. [12]. Figure 1(b) shows the arrangement of magnetic moments in such a phase. The calculated specific heat $C$ at constant volume is shown in the first panel of Fig. 2(a). It shows a typical $\lambda$ peak at $T_{\mathrm{AF}}$, characteristic of a continuous transition.

With a finite concentration of impurity bonds, $n_{\text {imp }}>0$, the peak in $C$ broadens, while its position remains almost constant as long as $n_{\text {imp }} \leq 0.04$. However, the magnitude of the collinear order parameter, $m_{\mathrm{AF}}$, shown in the second panel of Fig. 2(a), is strongly suppressed below $T=T_{\text {spi }} \simeq$ $150 \mathrm{~K}$ for $n_{\text {imp }}=0.02$. This suggests the onset of spiral order. Simultaneously, $\psi$ (estimated from $\sqrt{\left\langle\psi^{2}\right\rangle}$ ) becomes nonzero, and thus electric polarization sets in, provided the spiral plane is tilted out of the $a b$ plane, i.e., $\hat{\boldsymbol{n}}^{*} \wedge \hat{\boldsymbol{k}}_{\mathrm{sp}} \neq 0$. The associated susceptibility $\chi_{\psi}$ exhibits a peak, which seems to diverge as the system size increases, as shown in Ref. [26]. In Fig. 2(b), we show the spin-structure factor $S\left(q_{c}\right)$ as a function of the wave vector $q_{c}$ along the $c$ axis (averaged over $q_{a}$ and $q_{b}$ ) and temperature, for four values of $n_{\mathrm{imp}}$. At $n_{\mathrm{imp}}=0.02$, the propagation wave vector $q_{c}$ of the magnetic order decreases smoothly from $\pi$ below $T_{\text {spi, }}$, suggesting a continuous transition from the antiferromagnetic phase to a spiral-ordered phase, consistent with the experimental observations reported in Ref. [12]. A small residual $m_{\mathrm{AF}}$ below $T_{\text {spi }}$ remains. This might be due to either finite-size effects or a coexistence of the spiral and antiferromagnetic order [26]. Figure 2(c) shows $T_{\mathrm{spi}}$, estimated from the peak of $\chi_{\psi}$ (see Ref. [26]), as a function
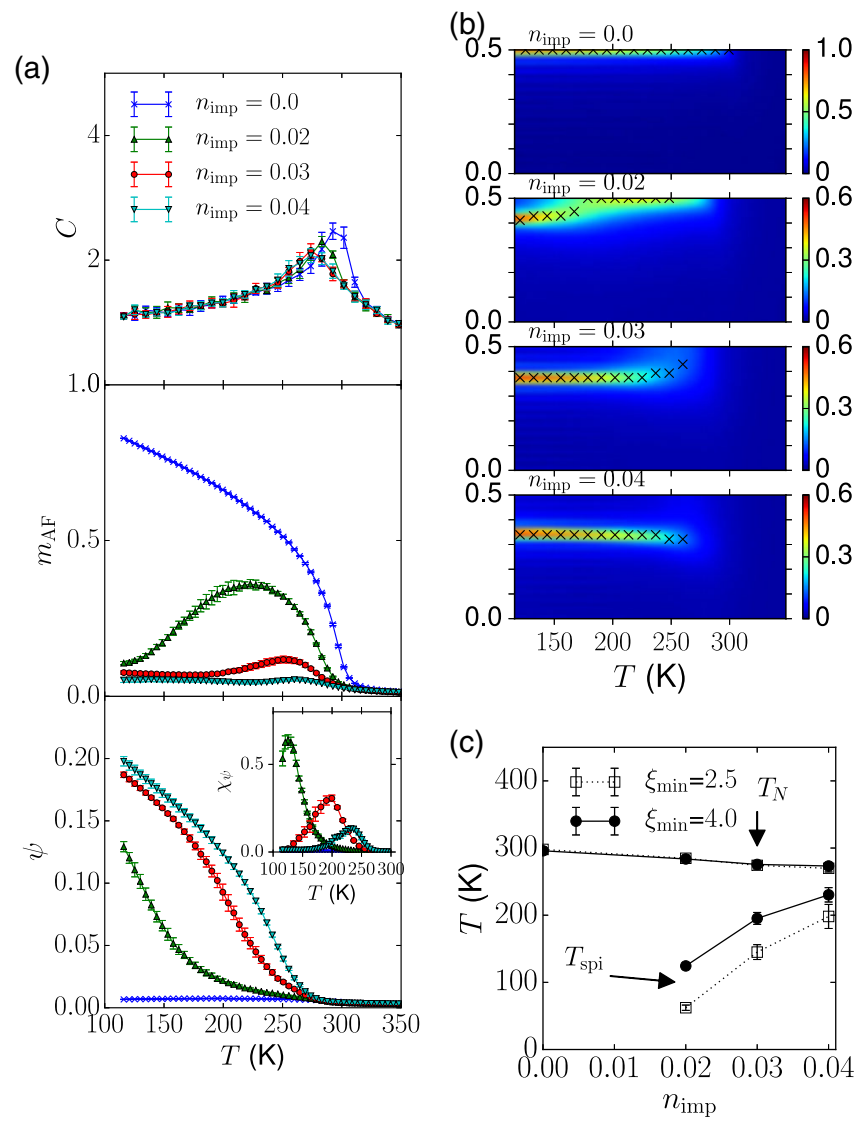

FIG. 2. Monte Carlo results obtained for systems of size $L=28$. (a) Specific heat $C$, collinear antiferromagnetic order parameter $m_{\mathrm{AF}}$, and spiral order parameter $\psi$, which we estimated by computing $\sqrt{\left\langle\psi^{2}\right\rangle}$, for $\xi_{\text {min }}=4$ and a range of impurity bond concentrations. (b) Square root of the spin-structure factor $S\left(q_{c}\right)$ computed for $\xi_{\min }=4$. The data are averaged over the in-plane wave vectors. The crosses represent the wave vector at which $S\left(q_{c}\right)$ takes a maximum value at the given temperature. The data for $n_{\text {imp }}=0.04$ are compatible with a direct transition from the paramagnetic state into an incommensurate magnetic state with $q_{\text {spi }}<1 / 2$. (c) Phase diagrams obtained for $\xi_{\min }=2.5$ and $\xi_{\min }=4$.

of impurity concentration. At large impurity concentration, $n_{\text {imp }} \gtrsim 0.04$, a direct transition from the paramagnet to an incommensurate spiral state with the spiral ordering wave vector $\boldsymbol{q}_{\mathrm{spi}}:=\left(1 / 2,1 / 2, q_{\mathrm{spi}}\right)$, whereby $q_{\mathrm{spi}}<1 / 2$, is compatible with the finite resolution of our data.

We carried out Monte Carlo simulations for the two values $\xi_{\min }=2.5$ and $\xi_{\min }=4$ of the minimal in-plane distance between impurity bonds for each value of $n_{\text {imp }}$. As $n_{\text {imp }}$ increases, $T_{\text {spi }}$ increases and almost reaches $T_{\mathrm{AF}}$ (as estimated from the peak in specific heat) at $n_{\text {imp }}=0.04$. Note that at $n_{\text {imp }}=0.04, S\left(q_{c}\right)$ has a maximum at $q_{c}<\pi$ even at high temperatures. This behavior does not rule out a direct transition from the paramagnetic to the spiral phase for larger (but not too large) values of $n_{\text {imp. Note that our }}$ values of $T_{\text {spi }}$ constitute lower bounds due to finite-size 
effects in the Monte Carlo simulations. Indeed, the peak values for $\chi_{\psi}$ are still moving to higher temperatures for the largest linear system sizes $L$ that were used in the Monte Carlo simulations. Hence, the finite-size effects on $T_{\text {spi }}$ are expected to be sizable for the values of $L$ used in our Monte Carlo simulations.

Finally, we discuss the short-range repulsion among the impurity bonds, which we model by imposing $\xi_{\text {min }}$. We find that when we do not include this effect, by setting $\xi_{\min }=0$, the low-temperature state does not support any spiral order over the range $0.02 \leq n_{\text {imp }} \leq 0.04$ that we studied. Instead, a "fan state" in which the intralayer ferromagnetic order oscillates is stabilized, as shown in panel (c) of Fig. 4. The fan state is discussed in more detail in Ref. [27]. Comparing the phase diagrams obtained for $\xi_{\min }=4$ and $\xi_{\min }=2.5$ in Fig. 2, we see that $T_{\text {spi }}$ decreases with decreasing $\xi_{\min }$. Indeed, the spiral order is favored if the presence of impurity bonds is suppressed in directions forming small angles with the $c$ axis, where the coupling has antiferromagnetic sign.

In summary, our simulations reveal that, in the range $0.02 \leq n_{\text {imp }} \leq 0.04$, the low-temperature state is a spiral with a temperature-dependent wave vector, provided the impurity bonds obey the condition that $\xi_{\min } \gtrsim 2.5$. Both the spiral ordering temperature $T_{\text {spi }}$ and the component $1 / 2-\boldsymbol{q}_{\mathrm{spi}} \cdot \hat{\boldsymbol{c}}$ of the ordering wave vector depend on the concentration $n_{\mathrm{imp}}$ of the impurity bonds and are proportional to the latter in the limit of small $n_{\text {imp. }}$.

\section{MECHANISM FOR SPIRAL STABILIZATION}

We now analyze the limit of low concentration $n_{\text {imp }} \ll 1$ of impurity bonds $n_{\text {imp }}$ and low temperature $T$. We show that, in this limit, antiferromagnetism can give way to a spiral order. For $T<T_{\mathrm{AF}}$, the expectation values for the spins become coplanar, and the common plane is determined by spin-orbit interactions. In the simplest case of Eq. (2), the magnetization will lie in the $a b$ plane. Thermal fluctuations out of the plane are not essential to the formation of the spiral, their main effect being a renormalization of the couplings between the in-plane components. For $n_{\text {imp }}=0$, the antiferromagnetic ground state has all spins parallel in the $x y$ plane, e.g., parallel to the $x$ axis. A single impurity bond with exchange of magnitude $\left|J_{\text {imp }}\right|$ above a threshold value $J_{\mathrm{c}}$ renders the ground state twofold degenerate (see Fig. 3). This fact was first explained by Villain in Ref. [20]. In Ref. [27], we applied the Villain model to show that, in this case, $J_{\mathrm{c}} \approx J_{\|} /\left[C-\left(\ln \left|J_{\perp}^{\prime} / J_{\|}\right|\right) /(2 \pi)\right]$, with $C \sim 0.4$ for $\left|J_{\perp}^{\prime} / J_{\|}\right| \ll 1$. Far away from the impurity bond, the staggered magnetization parallel to the $x$ axis is restored. Close to the impurity bond, which we label with the site $\tilde{\boldsymbol{r}}$ at its lower end, the coplanar spins are canted away from the $x$ axis, with the two ground states differing in the local sense of rotation of spins, as may be best characterized by [compare with Eq. (3)]

$$
\psi_{\mathrm{loc}}(\tilde{\boldsymbol{r}}):=\left(\mathbf{S}_{\tilde{\boldsymbol{r}}} \wedge \mathbf{S}_{\tilde{\boldsymbol{r}}+\hat{z}}\right) \cdot \hat{\boldsymbol{c}} .
$$

At finite but small $n_{\text {imp }}>0$, the impurity bonds are well separated. Thus, the low-energy configurations can be labeled by local Ising variables,

$$
\sigma_{\tilde{\boldsymbol{r}}}:=\operatorname{sgn} \psi_{\mathrm{loc}}(\tilde{\boldsymbol{r}}),
$$

describing the sign of the local cantings, while the remaining degrees of freedom are well captured by spin waves. Integrating the spin waves out yields an effective Hamiltonian $H_{\text {eff }}$ for the Ising degrees of freedom,

$$
H_{\text {eff }}=-\frac{\alpha^{2}}{2}\left(\sum_{\tilde{r}, \tilde{r}^{\prime} \in \mathcal{L}} \Gamma_{\tilde{r}, \tilde{r}^{\prime}} \sigma_{\tilde{r}} \sigma_{\tilde{r}^{\prime}}+\frac{N\left|J_{\perp}\right|}{\alpha^{2}} Q^{2}\right),
$$

for a system with $N$ lattice sites, where we simplified the model slightly by setting $\left|J_{\perp}\right|=J_{\perp}^{\prime}$. Here, $Q$ is the saddlepoint value of the spiral wave vector, and it is proportional to the net value of the Ising spins, (a)

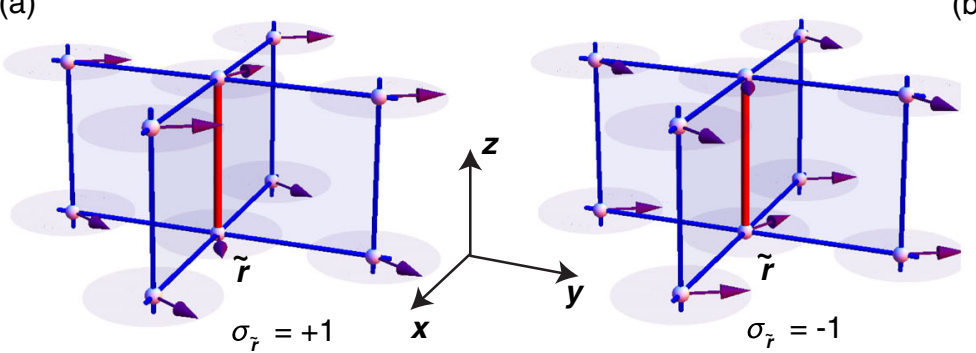

(b)

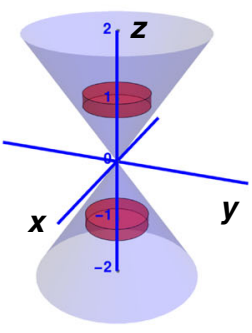

FIG. 3. (a) Sketch of the two ground states of Hamiltonian (1) for a single bilayer, in the presence of a single frustrating impurity bond (red line). For visualization purposes, the spins on every other site in the $a b$ plane have been reversed so that the unfrustrated ground state looks ferromagnetic. The two ground states break the local inversion symmetry and correspond to counterclockwise (left panel) and clockwise (right panel) rotation of the spins as one proceeds along the $c$ axis. They can be labeled by an Ising variable $\sigma_{\tilde{r}}$. Spin waves mediate an effective interaction $\Gamma_{\tilde{\boldsymbol{r}}, \tilde{r}^{\prime}}$ between the local orientations $\sigma_{\tilde{\boldsymbol{r}}}$ and $\sigma_{\tilde{\boldsymbol{r}}^{\prime}}$ of the cantings around two impurity bonds $\langle\tilde{\boldsymbol{r}}, \tilde{\boldsymbol{r}}+\boldsymbol{z}\rangle$ and $\left\langle\tilde{\boldsymbol{r}}^{\prime}, \tilde{\boldsymbol{r}}^{\prime}+\boldsymbol{z}\right\rangle$, as given by Eq. (6). (b) The blue cone is the domain of $\Delta \tilde{\boldsymbol{r}}=\tilde{\boldsymbol{r}}-\tilde{\boldsymbol{r}}^{\prime}$ for which the effective interaction $\Gamma_{\tilde{\boldsymbol{r}}, \tilde{r}^{\prime}}$ is antiferromagnetic, while outside the cone, it is ferromagnetic. The short-range constraint $\xi_{\min }$ excludes the domains represented by the red cylinders and thus increases the probability of a ferromagnetic coupling between neighboring Ising variables. 


$$
Q:=-\frac{|\alpha|}{N\left|J_{\perp}\right|} \sum_{\tilde{r} \in \mathcal{L}} \sigma_{\tilde{r}}
$$

where $\mathcal{L}$ denotes the ensemble of the impurity bonds in a given realization. Here, $Q$ is nonzero, provided the ground state of the effective Ising model has a net magnetization. In Eq. (6),

$$
\alpha:=\left(\left|J_{\text {imp }}\right|+\left|J_{\|}\right|\right)\left|\psi_{\text {loc }}^{\text {imp }}\right|,
$$

where $\psi_{\text {loc }}^{\mathrm{imp}}$ encodes the canting angle at an isolated impurity bond [27]. As shown in Ref. [27], at large distances, the kernel $\Gamma$ takes the (anti)dipolar form [28]

$$
\Gamma_{\tilde{r}, \tilde{r}^{\prime}} \sim \frac{\sqrt{\left|J_{\|}\right|}}{4 \pi} \frac{\left|J_{\perp}\right|\left(\Delta \tilde{r}_{x}^{2}+\Delta \tilde{r}_{y}^{2}\right)-2\left|J_{\|}\right| \Delta \tilde{r}_{z}^{2}}{\left[\left|J_{\perp}\right|\left(\Delta \tilde{r}_{x}^{2}+\Delta \tilde{r}_{y}^{2}\right)+\left|J_{\|}\right| \Delta \tilde{r}_{z}^{2}\right]^{\frac{5}{2}}} .
$$

Note that $\Gamma_{\tilde{r}, \tilde{r}^{\prime}}$ is ferromagnetic if $\Delta \tilde{r}_{x}^{2}+\Delta \tilde{r}_{y}^{2}>$ $\sqrt{2}\left|J_{\|}\right| \Delta \tilde{r}_{z}^{2} /\left|J_{\perp}\right|$ and antiferromagnetic otherwise, as illustrated in Fig. 3(b). In particular, the ratio of the in-plane nearest-neighbor interaction to the interplane one scales like $\left(J_{\|} / J_{\perp}\right)^{3 / 2} \gg 1$. We thus expect ferromagnetic order in plane. The interlayer order might be either ferromagnetic or antiferromagnetic. Now, the Coulomb repulsion between $\mathrm{Fe}^{3+} / \mathrm{Fe}^{3+}$ bipyramids amounts to a suppression of antiferromagnetically coupled pairs of nearest-neighbor impurities. A mean-field calculation shows that this constraint stabilizes ferromagnetic interlayer order. In the numerical simulation, we retain the essential part of the short-distance repulsion by forbidding impurity bonds on adjacent bilayers to be closer than $\xi_{\text {min }}$ in plane. Increasing $\xi_{\text {min }}$ indeed enhances the tendency toward a ferromagnetic Ising ground state with a finite $Q=n_{\text {imp }}\left|\alpha / J_{\perp}\right|$ (as opposed to competing interlayer antiferromagnetic order, which has vanishing $Q$ ).

The finite value of $Q$ immediately translates into a spiral order of the spins $S_{r}$, with the characteristic wave vector $\boldsymbol{q}_{\mathrm{spi}}=(1 / 2,1 / 2,1 / 2-Q)$. The linear dependence of $Q$ on $n_{\text {imp }}$ is in qualitative agreement with the $n_{\text {imp }}$ dependence of the peak of the low- $T$ structure factor, as calculated with Monte Carlo simulations and presented in Fig. 2(b).

The ground state of the effective Ising Hamiltonian (6) can be solved analytically when the set of impurity bonds forms a superlattice. Following Ref. [27], we assume that the impurity bonds form a Bravais lattice with the basis $\boldsymbol{A}, \boldsymbol{B}$, and $\boldsymbol{C}$ [29], such that $n_{\text {imp }}=\{[\boldsymbol{a} \cdot(\boldsymbol{b} \wedge \boldsymbol{c})] / \boldsymbol{A} \cdot(\boldsymbol{B} \wedge \boldsymbol{C})\} \ll 1$. In Figs. 4(a) and 4(b), we compare the ground state of Eq. (6a) (a)

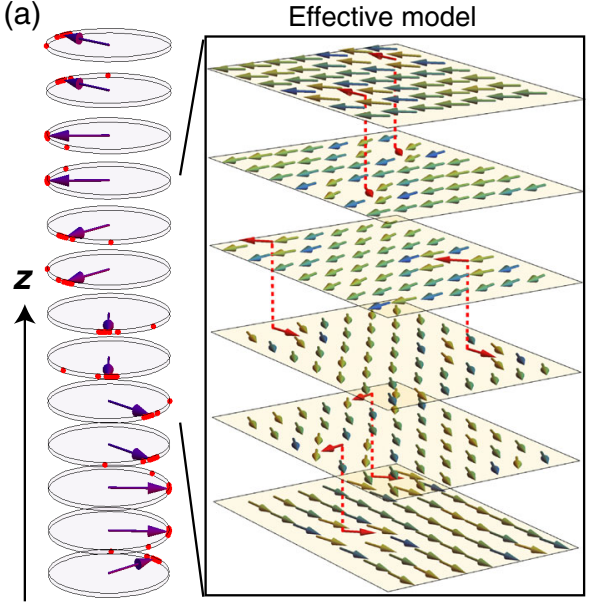

Numerical minimization

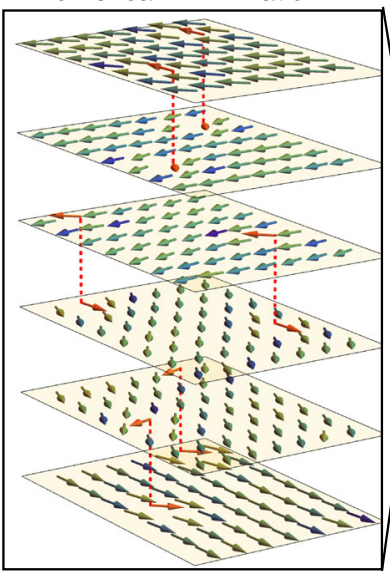

(b)

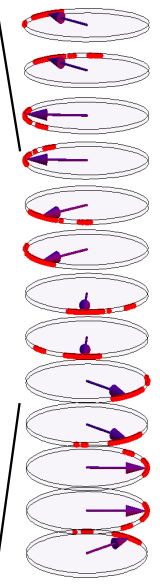

(d) (c)

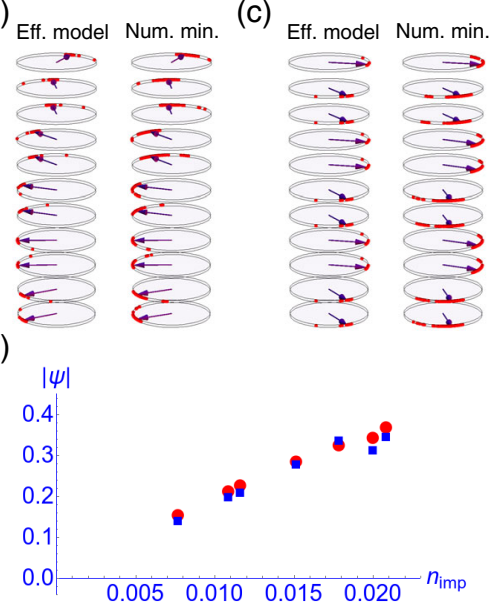

FIG. 4. (a) Comparison of a spiral ground state corresponding to an Ising ferromagnetic ground state of the effective Ising Hamiltonian (6) (left) against the spiral ground state of the Heisenberg Hamiltonian (1) (right). The violet arrows represent the average directions of the staggered magnetization for each layer. The red dots lying on the circle represent the direction of each spin in a given plane. The staggered magnetization of layers corresponding to even bilayers have been reversed for visualization purposes. The two central panels depict the ground states in a portion of six layers of the lattice of overall dimensions $14 \times 14 \times 32$. Here, the color of the arrows indicates the angular difference of each spin from the average staggered magnetization of the layer, and the vertical red lines depict the impurity bonds. For visualization purposes, spins at every other site in the $a b$ plane and at every other bilayer have been reversed. The results were obtained for a superlattice of impurity bonds with lattice constants $\boldsymbol{A}=(4,3,0), \boldsymbol{B}=(0,4,2)$, and $\boldsymbol{C}=(4,0,2)$. The values of the couplings are $J_{\|}=28.9 \mathrm{meV}, J_{\perp}=J_{\perp}^{\prime}=4.1 \mathrm{meV}$, and $J_{\text {imp }}=-95.8 \mathrm{meV}$. (b) Comparison of the spiral ground state obtained using the effective Ising Hamiltonian (6) (left) against the spiral ground state of the Heisenberg Hamiltonian (1) (right) for the same value of the couplings but with $\boldsymbol{A}=(5,3,2), \boldsymbol{B}=(3,0,4)$, and $\boldsymbol{C}=(2,4,6)$. Panel (c) shows the same comparison for the choice $\boldsymbol{A}=(5,0,0)$, $\boldsymbol{B}=(0,5,0)$, and $\boldsymbol{C}=(0,1,2)$. For such a superlattice of impurity bonds, a fan state (with no net winding along the $c$ axis) is stabilized instead of a spiral. (d) Concentration dependence of the magnitude of $\psi$ defined in Eq. (3) for the ground states obtained from the effective Ising Hamiltonian (6) (red dots) and the Heisenberg Hamiltonian (1) (right) (blue squares) for various superlattices of impurity bonds stabilizing a spiral state. 
with the ground state obtained by Monte Carlo simulations of Eq. (1). For both calculations, we take $\left|J_{\perp}^{\prime}\right|=\left|J_{\perp}\right|=$ $4.1 \mathrm{meV}$ to be the average of the actual $\left|J_{\perp}\right|$ and $\left|J_{\perp}^{\prime}\right|$ given in Fig. 1. In agreement with the magnetic structure obtained by the refined analysis of the elastic neutron-scattering data, the obtained magnetic spiral has the feature that the rotation of magnetic moments mainly happens between neighboring $a b$ layers coupled by impurity bonds (e.g., bilayers of the $\mathrm{YBaCuFeO}_{5}$ structure).

Figure 4(c) gives a similar comparison but for a superlattice favoring a state with no net winding of the spins, i.e., a fanlike magnetic state.

Finally, Fig. 4(d) shows the concentration dependence of the spiral order parameter $\psi$ [recall Eq. (3)], calculated both numerically from Eq. (1) and using the effective Ising Hamiltonian (6) for various superlattices for which the ground state is a spiral. The values of $\psi$ predicted by the effective model match well with the values of $\psi$ obtained numerically for the ground state of a finite system. Furthermore, the value of $\psi$ increases proportionally with $n_{\text {imp }}$ for small values of $\psi$ as predicted by the effective model.

As we have already mentioned, the addition of spin-orbit interactions to the Heisenberg model lifts the degeneracy of spiral states that differ by the orientation of their spiral planes. The strongest spin-orbit terms are expected to be Dzyaloshinskii-Moriya (DM) terms of the form $\boldsymbol{D}_{i j} \cdot \boldsymbol{S}_{i} \wedge \boldsymbol{S}_{j}$, as the couplings $\boldsymbol{D}_{i j}$ are of first order in the weak spin-orbit coupling parameter, in contrast to single ion anisotropies, which are of second order and thus parametrically weaker. DM terms are most relevant on bonds close to defects. This is so because, on the one hand, the canting in their vicinity leads to an appreciable value of $S_{i} \wedge S_{j}$, while, on the other hand, the couplings $D_{i j}$ are expected to be stronger in that region since defects locally distort the lattice, reducing the symmetry of the bond's environment, which is often needed to obtain a nonvanishing DM coupling. The dominant DM terms favor tilting the spiral plane with respect to the $a b$ plane, while the average single-ion anisotropy of the bulk empirically seems to prefer an in-plane magnetization. For a large-enough magnitude of the DM terms, the competition between the two terms will induce a finite inclination of the spiral plane, which monotonically increases with defect concentration, in qualitative accordance with what was reported in Refs. $[12,16]$. Even at very low defect concentration, this mechanism makes the spiral state acquire a cycloidal component, which permits coupling between the magnetic order and electric polarization. The details of this mechanism will be discussed elsewhere.

\section{CONCLUSIONS}

We have presented a mechanism in which impurity bonds, when sufficiently strong, induce a local frustration in an otherwise nonfrustrated lattice of classical spins. The associated cantings become long-range correlated at low temperature, resulting in a spiral magnetic order in which the sense of rotation of the spiral is spontaneously chosen. The spiral formation is caused by orientational long-range correlations, which align the impurity bonds along one crystallographic direction of the lattice, thus avoiding the spin-glass phase expected for white-noise correlated random magnetic exchange interactions [18-21,30]. The critical temperature below which the spiral order develops is controlled by nearest-neighbor exchange couplings, which can be sizable, so the mechanism is relevant for engineering high-temperature multiferroics.

When the model is applied to $\mathrm{YBaCuFeO}_{5}$ with realistic couplings, it predicts a multiferroic phase that is consistent with several distinct features observed experimentally. (i) It captures the transition to a commensurate antiferromagnet phase with wave vector $\boldsymbol{q}_{\mathrm{AF}} \equiv(1 / 2,1 / 2,1 / 2)$ at the Néel temperature $T_{\mathrm{AF}}$, followed by a transition to an incommensurate spiral phase with wave vector $\boldsymbol{q}_{\mathrm{spi}} \equiv$ $(1 / 2,1 / 2,1 / 2-Q)$ at the lower critical temperature $T_{\text {spi }}$. (ii) It gives rise to a magnetic spiral phase, in which the rotation of the magnetic moments neighboring along $c$ occurs primarily when they belong to the same bilayer. (iii) The observed temperature dependence of $Q$ in $\mathrm{YBaCuFeO}_{5}$ is well reproduced. (iv) Finally, the dependence of both $T_{\text {spi }}$ and $\boldsymbol{q}_{\text {spi }}$ on the concentration $n_{\text {imp }}$ is in qualitative agreement with the dependence on the annealing conditions of the $\mathrm{YBaCuFeO}_{5}$ samples: The faster the quench (and thus the higher the expected defect concentration), the larger the measured $T_{\text {spi }}$ and $\boldsymbol{q}_{\text {spi }}$.

We close by mentioning other compounds with magnetic spiral order whose origin is not understood so far but to which the mechanism presented in this paper might apply. First, we mention the numerical results obtained in the model twodimensional systems studied by Ivanov et al. [31] and by Capati et al. [32]. There, too, impurity bonds were orientationally correlated, and a related mechanism was likely at play. For solid solutions of $\mathrm{Cr}_{2} \mathrm{O}_{3}$ and $\mathrm{Fe}_{2} \mathrm{O}_{3}$ [33] and for the doped hexaferrite $\mathrm{Ba}_{(1-x)} \mathrm{Sr}_{x} \mathrm{Zn}_{2} \mathrm{Fe}_{12} \mathrm{O}_{22}$ [34], the wave vector of the spiral order is known to change smoothly from a commensurate value at high temperatures to an incommensurate value at low temperatures. In all cases, the maximal value of the concentration-dependent $T_{\text {spi }}$ is high ( $T_{\text {spi }}^{\max }=148 \mathrm{~K}$ for solid solutions of $\mathrm{Cr}_{2} \mathrm{O}_{3}$ and $\mathrm{Fe}_{2} \mathrm{O}_{3}$ [33] and $T_{\text {spi }}^{\max }$ higher than $294 \mathrm{~K}$ for $\mathrm{Ba}_{(1-x)} \mathrm{Sr}_{x} \mathrm{Zn}_{2} \mathrm{Fe}_{12} \mathrm{O}_{22}$ [35]). Moreover, the wave vector and the transition temperature to the spiral state are dependent on the dopant concentration. Since in both compounds cation substitution can introduce impurity bonds, they are likely candidates to realize "spiral order by disorder."

\section{ACKNOWLEDGMENTS}

This research was partially supported by NCCR MARVEL, funded by the Swiss National Science 
Foundation. H. S. acknowledges support from the DFG via FOR 1346, the SNF Grant No. 200021E-149122, ERC Advanced Grant SIMCOFE, and ERC Consolidator Grant CORRELMAT (Project No. 617196). Computer time was provided through a grant from the Swiss National Supercomputing Centre (CSCS) under Project No. ID p504, as well as by the Brutus cluster of ETH Zürich. We thank M. Kenzelmann, M. Medarde, and M. Morin for very useful discussions. H.S. was supported by JSPS KAKENHI Grants No. 16H01064 (J-Physics) and No. 16K17735.

[1] H. Katsura, N. Nagaosa, and A. V. Balatsky, Spin Current and Magnetoelectric Effect in Noncollinear Magnets, Phys. Rev. Lett. 95, 057205 (2005).

[2] M. Mostovoy, Ferroelectricity in Spiral Magnets, Phys. Rev. Lett. 96, 067601 (2006).

[3] D. Khomskii, Trend: Classifying Multiferroics: Mechanisms and Effects, Physics 2, 20 (2009).

[4] Y. Tokura and S. Seki, Multiferroics with Spiral Spin Orders, Adv. Mater. 22, 1554 (2010).

[5] T. Kimura, Spiral Magnets as Magnetoelectrics, Annu. Rev. Mater. Res. 37, 387 (2007).

[6] T. Kimura, T. Goto, H. Shintani, K. Ishizaka, T. Arima, and Y. Tokura, Magnetic Control of Ferroelectric Polarization, Nature (London) 426, 55 (2003).

[7] T. Goto, T. Kimura, G. Lawes, A. P. Ramirez, and Y. Tokura, Ferroelectricity and Giant Magnetocapacitance in Perovskite Rare-Earth Manganites, Phys. Rev. Lett. 92, 257201 (2004).

[8] M. Kenzelmann, A. B. Harris, S. Jonas, C. Broholm, J. Schefer, S. B. Kim, C. L. Zhang, S.-W. Cheong, O. P. Vajk, and J. W. Lynn, Magnetic Inversion Symmetry Breaking and Ferroelectricity in $\mathrm{TbMnO}_{3}$, Phys. Rev. Lett. 95, 087206 (2005).

[9] S. Dong, R. Yu, S. Yunoki, J.-M. Liu, and E. Dagotto, Origin of Multiferroic Spiral Spin Order in the $R \mathrm{MnO}_{3}$ Perovskites, Phys. Rev. B 78, 155121 (2008).

[10] M. Mochizuki and N. Furukawa, Microscopic Model and Phase Diagrams of the Multiferroic Perovskite Manganites, Phys. Rev. B 80, 134416 (2009).

[11] B. Kundys, A. Maignan, and C. Simon, Multiferroicity with High- $T_{C}$ in Ceramics of the $\mathrm{YBaCuFeO}_{5}$ Ordered Perovskite, Appl. Phys. Lett. 94, 072506 (2009).

[12] M. Morin, A. Scaramucci, M. Bartkowiak, E. Pomjakushina, G. Deng, D. Sheptyakov, L. Keller, J. Rodriguez-Carvajal, N. A. Spaldin, M. Kenzelmann, K. Conder, and M. Medarde, Incommensurate Magnetic Structure, $\mathrm{Fe} / \mathrm{Cu}$ Chemical Disorder, and Magnetic Interactions in the High-Temperature Multiferroic $\mathrm{YBaCuFeO}_{5}$, Phys. Rev. B 91, 064408 (2015).

[13] V. Caignaert, I. Mirebeau, F. Boure, N. Nguyen, A. Ducouret, J.-M. Greneche, and B. Raveau, Crystal and Magnetic Structure of $\mathrm{YBaCuFeO}_{5}$, J. Solid State Chem. 114, 24 (1995).

[14] Y. Kawamura, T. Kai, E. Satomi, Y. Yasui, Y. Kobayashi, M. Sato, and K. Kakurai, High-Temperature Multiferroic State of $\mathrm{RBaCuFeO}_{5}(\mathrm{R}=\mathrm{Y}, L u$, and Tm), J. Phys. Soc. Jpn. 79, 073705 (2010).

[15] M. J. Ruiz-Aragon, E. Moran, U. Amador, J. L. Martinez, N.H. Andersen, and H. Ehrenberg, Low-Temperature Magnetic Structure of $\mathrm{YBaCuFeO}_{5}$ and the Effect of Partial Substitution of Yttrium by Calcium, Phys. Rev. B 58, 6291 (1998).

[16] M. Morin, E. Canévet, A. Raynaud, M. Bartkowiak, D. Sheptyakov, B. Voraksmy, M. Kenzelmann, E. Pomjakushina, K. Conder, and M. Medarde, Tuning Magnetic Spiral Beyond Room Temperature with Chemical Disorder, Nat. Commun. 7, 13758 (2016).

[17] Note that the parameters used in this paper differ slightly from those of Ref. [12] as the LSDA $+U$ method used to extract them in Ref. [12] differs from the one used here.

[18] S. F. Edwards and P. W. Anderson, Theory of Spin Glasses, J. Phys. F 5, 965 (1975).

[19] K. Binder and A. P. Young, Spin Glasses: Experimental Facts, Theoretical Concepts, and Open Questions, Rev. Mod. Phys. 58, 801 (1986).

[20] J. Villain, Two-Level Systems in a Spin-Glass Model. I. General Formalism and Two-Dimensional Model, J. Phys. C 10, 4793 (1977).

[21] J. Villain, Two-Level Systems in a Spin-Glass Model: II. Three-Dimensional Model and Effect of a Magnetic Field, J. Phys. C 11, 745 (1978).

[22] The antiferromagnetic $\mathrm{Fe}^{3+}-\mathrm{Fe}^{3+}$ coupling ranges between $90 \mathrm{meV}$ and $94 \mathrm{meV}$ in magnitude depending on the neighboring bipyramids. (We use the convention that the magnetic moments $|\boldsymbol{S}|$ are of unit length.) Ab initio calculations were done using the same method as in Ref. [12].

[23] The magnetic exchange couplings have been rescaled so as to account for the magnitude of the classical magnetic moments of the ions located on the sites $\boldsymbol{r}$ and $\boldsymbol{r}^{\prime}$.

[24] Y.-C. Lai, C.-H. Du, C.-H. Lai, Y.-H. Liang, C.-W. Wang, K. C. Rule, H.-C. Wu, H.-D. Yang, W.-T. Chen, G. J. Shu, and F.-C. Chou, Magnetic Ordering and Dielectric Relaxation in the Double Perovskite $\mathrm{YBaCuFeO}_{5}$, J. Phys. Condens. Matter 29, 145801 (2017).

[25] N. Nagaosa, Theory of Multiferroic Behavior in Cycloidal Helimagnets, J. Phys. Condens. Matter 20, 434207 (2008).

[26] See Supplemental Material at http://link.aps.org/ supplemental/10.1103/PhysRevX.8.011005 for more details on Monte Carlo simulations and results.

[27] A. Scaramucci, H. Shinaoka, M. V. Mostovoy, M. Müller, and C. Mudry, Spiral Order from Orientationally Correlated Random Bonds in Classical XY Models, arXiv: 1610.00784.

[28] Note that the kernel Eq. (6d) encodes the effective coupling between the sense of rotation of the canting at two impurity bonds. Despite the dipolar form of these couplings, the canting patterns should not be interpreted as carrying an electric or magnetic dipole.

[29] We choose $\boldsymbol{A}, \boldsymbol{B}$, and $\boldsymbol{C}$ such that the impurity bonds are confined to the bilayers of the host lattice as in $\mathrm{YBaCuFeO}_{5}$.

[30] J. Villain, Theory of One- and Two-Dimensional Magnets with an Easy Magnetization Plane. II. The Planar, 
Classical, Two-Dimensional Magnet, J. Phys. (Paris) 36, 581 (1975).

[31] N. B. Ivanov, S. E. Kruger, and J. Richter, Square-Lattice Heisenberg Antiferromagnet with Two Kinds of NearestNeighbor Regular Bonds, Phys. Rev. B 53, 2633 (1996).

[32] M. Capati, S. Caprara, C. Di Castro, M. Grilli, G. Seibold, and J. Lorenzana, Electronic Polymers and Soft-Matter-Like Broken Symmetries in Underdoped Cuprates, Nat. Commun. 6, 7691 (2015).
[33] D. E. Cox, W. J. Takei, and G. Shirane, A Magnetic and Neutron Diffraction Study of the $\mathrm{Cr}_{2} \mathrm{O}_{3}-\mathrm{Fe}_{2} \mathrm{O}_{3}$ System, J. Phys. Chem. Solids 24, 405 (1963).

[34] S. Utsumi, D. Yoshiba, and N. Momozawa, Superexchange Interactions of $\left(\mathrm{Ba}_{1-x} \mathrm{Sr}_{x}\right)_{2} \mathrm{Zn}_{2} \mathrm{Fe}_{12} \mathrm{O}_{22}$ System Studied by Neutron Diffraction, J. Phys. Soc. Jpn. 76, 034704 (2007).

[35] N. Momozawa, Y. Yamaguchi, H. Takei, and M. Mita, Magnetic Structure of $\left(\mathrm{Ba}_{1-x} \mathrm{Sr}_{x}\right)_{2} \mathrm{Zn}_{2} \mathrm{Fe}_{12} \mathrm{O}_{22}(\mathrm{x}=0-1.0)$, J. Phys. Soc. Jpn. 54, 771 (1985). 